\title{
Influence spread in two-layer interdependent networks: designed single-layer or random two-layer initial spreaders?
}

\author{
Hana Khamfroush ${ }^{1}$, Nathaniel Hudson ${ }^{1 *}$ (D), Samuel loo $^{1}$ and Mahshid R. Naeini ${ }^{2}$
}

\author{
${ }^{*}$ Correspondence: \\ nathaniel.hudson@uky.edu \\ 'Department of Computer Science, \\ University of Kentucky, University of \\ Kentucky, Lexington 40506, KY, USA \\ Full list of author information is \\ available at the end of the article
}

\begin{abstract}
Influence spread in multi-layer interdependent networks (M-IDN) has been studied in the last few years; however, prior works mostly focused on the spread that is initiated in a single layer of an M-IDN. In real world scenarios, influence spread can happen concurrently among many or all components making up the topology of an M-IDN. This paper investigates the effectiveness of different influence spread strategies in M-IDNs by providing a comprehensive analysis of the time evolution of influence propagation given different initial spreader strategies. For this study we consider a two-layer interdependent network and a general probabilistic threshold influence spread model to evaluate the evolution of influence spread over time. For a given coupling scenario, we tested multiple interdependent topologies, composed of layers $A$ and $B$, against four cases of initial spreader selection: (1) random initial spreaders in $A$, (2) random initial spreaders in both $A$ and $B$, (3) targeted initial spreaders using degree centrality in $A$, and (4) targeted initial spreaders using degree centrality in both $A$ and $B$. Our results indicate that the effectiveness of influence spread highly depends on network topologies, the way they are coupled, and our knowledge of the network structure - thus an initial spread starting in only A can be as effective as initial spread starting in both $A$ and $B$ concurrently. Similarly, random initial spread in multiple layers of an interdependent system can be more severe than a comparable initial spread in a single layer. Our results can be easily extended to different types of event propagation in multi-layer interdependent networks such as information/misinformation propagation in online social networks, disease propagation in offline social networks, and failure/attack propagation in cyber-physical systems.
\end{abstract}

Keywords: Influence spread, Phenomena propagation, Information diffusion, Initial spreader selection, Seed selection, Multi-layer networks, Interdependent networks, Social networks, Cyber-physical systems

\section{Introduction}

Multi-layer interdependent networks (M-IDNs) are systems composed of more than one network with edges between them to form an interconnected environment. M-IDNs can be used to model many real-world interdependent systems, such as cyber-physical systems and online-offline social networks. In recent years, we have seen an exponential growth in the development and deployment of these systems. This growth is largely due

(c) The Author(s). 2019 Open Access This article is distributed under the terms of the Creative Commons Attribution 4.0 International License (http://creativecommons.org/licenses/by/4.0/), which permits unrestricted use, distribution, and reproduction in any medium, provided you give appropriate credit to the original author(s) and the source, provide a link to the Creative Commons license, and indicate if changes were made. 
to the increasing use of more technologies interfacing with one another - such as the Internet of Things. The explosive expansion and scale of technologies that connect with one another through the Internet provide further incentive to explore the interconnection of different real-world systems and their impacts on phenomena propagation in these environments.

The interdependency between separate networks creates specific characteristics for these systems that need to be investigated. Specifically, the interaction between separate networks opens up potential for unique opportunities to design new selection strategies that might not be as effective in a system made up of a single network. For example, the attack that was launched by Stuxnet (Albright et al. 2010) in 2010 was the result of a malicious computer worm that led to substantial damages to the programmable logic controller (PLC) systems and the power plants. The main reason for such wide-spread damage was the interdependency between the supervisory control and data acquisition (SCADA) system controlling the nuclear enrichment plants and the enrichment plants. This huge impact would likely not have been possible without the interdependency between the cyber (controller) component and the physical component's performance.

Recently, research aimed at investigating wide-spread phenomena propagation in MIDNs focuses on designing resilient and robust M-IDN systems. There is a considerable amount of literature addressing the problem of modeling and analyzing the impact of interdependency between different interdependent systems (such as cyber-physical systems) and how to minimize phenomena propagation in these systems. Work investigating selection strategies of initial spreaders in M-IDN systems has been conducted (as discussed in Related Work section). However, these works consider less general propagation models like epidemic and independent cascade. Additionally, our work is particularly interested in investigating how the evolution of affliction over time and a variety of interconnectivity and layer-based selection strategies for initial spreaders compare, distinguishing our work from that in the literature.

In short, we will make the following contributions:

- We propose a modified version of the threshold-based phenomena propagation model that was initially proposed in (Khamfroush et al. 2016) for a general interdependent network and a single phenomenon, as a mathematical model to quantify the impact of different strategies for selecting initial spreaders for phenomena propagation in an M-IDN system.

- We perform extensive simulations to study the effectiveness of different types of spread initialization including random/designed single-layer initial spread and random/designed multi-layer initial spread in a M-IDN system.

- We provide guidelines on which network topologies and types of coupling between the networks provide faster propagation when facing different strategies for spread initialization. Due to the generality of the proposed model, our observations provide a useful framework for further investigation and design of robust and reliable M-IDN.

\section{Related work}

Various works explore different approaches to either minimizing or maximizing phenomena propagation in M-IDN systems, depending on the context of the problem. For instance, in social networks, one may want to maximize the spread of critical information 
(Kim et al. 2014; 2015; Kandhway and Kuri 2017) or minimize the propagation of misinformation (or "fake news") (Papanastasiou 2018; Kimura et al. 2009; Shu et al. 2019). Since our problem is general in nature, we do not consider a context that implies whether the phenomena propagation has positive or negative ramifications. We are simply interested in how certain selection strategies of initial spreaders affect overall phenomena propagation.

Securing an M-IDN goes beyond securing the separate networks composing the entire interdependent topology. Adversaries are willing to use the interdependency of vulnerabilities to carry out multi-stage attacks. Each facet of an attack may not pose a significant threat to a single network on its own; however, cumulative influences through interdependency, may have catastrophic effects.

In the past few years, vulnerabilities in M-IDNs have been widely studied. In general, there are two main approaches to study this vulnerability. The first approach typically involves focusing on a certain application of M-IDNs to identify different forms of attacks/threats and potential strategies to protect corresponding M-IDN systems. For example, (Anderson and Fuloria 2010; McDaniel and McLaughlin 2009; Metke and Ekl 2010; Mo et al. 2012; Rahman et al. 2012) looked into financially motivated threats in smart grids where a customer who wants to trick a utility company's billing system tampers with smart meters to reduce the electricity bill. Other studies, such as (Halperin et al. 2008; Hanna et al. 2011; Rushanan et al. 2014), looked into the threats against medical cyber-physical systems. Authors in (Brooks et al. 2008; Checkoway et al. 2011; Hoppe et al. 2008) defined and investigated different threats in smart cars as another application of cyber-physical systems.

In our prior work (Hudson et al. 2019), we investigate the effectiveness of standard and interdependent centrality metrics to minimize cascade of failure. In that work, we used random failure to select initially failed nodes to begin failure propagation. There was no investigation, in this prior work, into how different strategies for initial spread performed.

In the past, there have been studies conducted that investigate the effectiveness of selection strategies in singular networks (Albert et al. 2000; Pastor-Satorras and Vespignani 2001; Cohen et al. 2001). However, authors in (Salehi et al. 2015) provide a thorough overview of prior works investigating this topic for M-IDN networks. These prior works consider different models of phenomena propagation for the spread of a phenomenon through an M-IDN system. For example, (Erlandsson et al. 2017) investigates seed selection strategies to maximize information cascade in multi-layer social networks under an independent cascade model (ICM), where nodes across different layers are one and the same - so if a node $i_{A}$ is afflicted in layer $A$ from an intra-neighbor in that layer, node $i_{B}$ in layer $B$ is afflicted as an immediate result. This work concluded that degree centrality, out of the strategies considered by this work, is the overall most effective selection strategy for initial spreaders to maximize cascade in these environments. However, this work does not consider propagation over time as afflicted nodes only have one chance to afflict their neighbors. The work in (Zhao et al. 2014) considers an epidemic propagation model, where the goal is to identify the most influential nodes in the network. Also in the context of social networks, there have been a large body of work investigating the influence maximization 
problem that focuses on choosing optimal seed set such that the spread of information is maximized, e.g., (Michalski et al. 2014; Kempe et al. 2005; Chen et al. 2009; Chen et al. 2010).

In contrast to these prior works, our goal is not to find the most influential seed set, instead we are looking at the impact that different choices of the seed set (single-layer versus two-layer) could have on the propagation process. More specifically, we are investigating whether a single-layer seed selection could be as influential as a similar size two-layer seed selection. Furthermore, our study incorporates a more general propagation model and takes into consideration the evolution of the spread of a phenomenon over time. This consideration allows afflicted nodes more opportunities to afflict neighbor nodes, which is more realistic in many scenarios. For instance, in the context of information cascade in social networks one user may be afflicted by some information and share that with their friends. This person's friends may not immediately adopt the information that this person is propagating; however later in time this could change. The independent cascade model does not consider such cases and is thus inapplicable for many scenarios that involve delay. Further, linear threshold and linear probabilistic propagation are special use cases of our model.

\section{Problem statement}

We consider an M-IDN system consisting of two interdependent networks, A and B. Both layers $\mathrm{A}$ and $\mathrm{B}$ are represented by two undirected graphs $G_{A}=\left(V_{A}, E_{A}\right)$ and $G_{B}=\left(V_{B}, E_{B}\right)$, where $V_{A}$ and $V_{B}$ represent the sets of vertices in layers $A$ and $B$ (respectively) and $E_{A}$ and $E_{B}$ represent the sets of edges in layers $A$ and $B$ (respectively). It is assumed that $\left|V_{A}\right|=N_{A}$ and $\left|V_{B}\right|=N_{B}$. Though it is not contingent for $N_{A}$ to be equal to $N_{B}$, for our experiments $N_{A}=N_{B}$ in all synthetic cases. The two networks are interconnected by means of directed edges. We refer to edges that connect nodes belonging to the same networks as intra-edges and those that connect nodes belonging to different networks as inter-edges. We use directed edges for inter-edges to capture different models of inter-dependency between networks. Without loss of generality, it is assumed that an initial set $F_{0}=F_{0}^{A} \cup F_{0}^{B}$ of nodes are afflicted initially, where $F_{0}^{A}$ represents the set of initial spreaders in network $A$ and $F_{0}^{B}$ represents the set of initial spreaders in layer $B$. Phenomena can propagate among nodes belonging to the same network. In addition, phenomena can also propagate across networks, such as phenomena propagating from a node in layer $A$ to a node in layer $B$ (or vice-versa) through inter-edges. Phenomena propagation takes place with respect to a threshold-based propagation model - as discussed in more detail in "Phenomena propagation model" section. Given an M-IDN system with a known topology, we are interested in comparing the rate of phenomena propagation with respect to time under different types of initial spreads. More specifically, given an initial set of afflicted nodes $F_{0}$ ("initial spreaders"), we will evaluate phenomena propagation in an M-IDN system. The goal of this work is to provide useful guidelines on how to design reliable M-IDN systems. Additionally, we are particularly interested in the evolution of phenomena propagation in the case of single- and two-layer affliction.

To model different types of initial spreads in M-IDN systems, we will define different values for sets $F_{0}^{A}$ and $F_{0}^{B}$ and different selection strategies for these sets. More specifically, we analyze the following four types of initial spreads in an M-IDN. 
- Random single-layer spread is represented by defining $\left|F_{0}^{A}\right|=\left|F_{0}\right|$ and $\left|F_{0}^{B}\right|=0$, where the nodes of $F_{0}^{A}$ are chosen randomly ${ }^{1}$.

- Random two-layer spread is represented by defining $\left|F_{0}^{A}\right|=\left|F_{0}\right| / 2$ and $\left|F_{0}^{B}\right|=\left|F_{0}\right| / 2$, where the nodes of $F_{0}^{A}$ and $F_{0}^{B}$ are chosen randomly.

- Designed single-layer spread is represented by choosing $\left|F_{0}\right|$ nodes belonging to layer A with highest degree $F_{0}^{A}$, and setting $\left|F_{0}^{B}\right|=0$.

- Designed two-layer spread is represented by choosing $\left|F_{0}\right| / 2$ nodes belonging to layer $A$ with highest degree $F_{0}^{A}$ and $\left|F_{0}\right| / 2$ nodes belonging to layer $B$ with highest degree $F_{0}^{B}$.

Due to the general nature of our problem, we are not seeking to differentiate negative and positive phenomena propagation. We are simply interested in how certain selection strategies for initial spreaders affect overall phenomena propagation. For this reason, we refer to nodes that have been affected by the phenomena propagation as "afflicted" and the process by which nodes are afflicted as "affliction". This language is used to avoid the contextual effects of phenomena propagation for different domains. For an overview of the notation defined throughout this paper, refer to Table 1.

\section{Phenomena propagation model}

Many threshold-based propagation models rely on deterministic behavior; wherein if a proportion of a node's neighbors are afflicted, then it will be afflicted definitively. While this approach is appropriate for epidemic phenomena, this approach to phenomena propagation is not as general and fails to consider cases with probabilistic propagation. Due to this limitation we incorporate a modified version of probabilistic threshold-based phenomena propagation used in our prior works (Khamfroush et al. 2016; Hudson et al. 2019). As in our prior works, we considered nodes belonging to the same network to have peer roles and thus are connected through undirected edges. We refer to the adjacency matrices that represent layers $A$ and $B$ as $M_{A A} \in\{0,1\}^{N_{A} \times N_{A}}$ and $M_{B B} \in\{0,1\}^{N_{B} \times N_{B}}$, respectively. The interconnectivity between layers $A$ and $B$ can be represented as interconnection adjacency matrices $M_{A B} \in\{0,1\}^{N_{A} \times N_{B}}$ and $M_{B A} \in\{0,1\}^{N_{B} \times N_{A}}-$ with the

Table 1 Summary table of noteworthy notation introduced

\begin{tabular}{ll}
\hline Symbol & Description \\
\hline$G_{A}=\left(V_{A}, E_{A}\right)$ & Graph for layer $A$. \\
$N_{A}$ & Number of nodes in layer $A$. \\
$F_{0}$ & Set of initial spreaders afflicted at the start of propagation. \\
$F_{0}^{A}$ & Set of initial spreaders in layer $A$ afflicted at the start of propagation. \\
$M_{A A}$ & Adjacency matrix representing layer $A$. \\
$M_{A B}$ & Adjacency matrix representing inter-edges directed from layer $A$ to layer $B$. \\
$U_{A}^{\text {intra }}(i)$ & Set of intra-neighbors for node $i$ in layer $A$. \\
$U_{A}^{\text {inter }}(i)$ & Set of inter-parents for node $i$ in layer $A$. \\
$\alpha(i)$ & Fraction of intra-neighbors of node $i$ that are afflicted. \\
$\beta(i)$ & Fraction of inter-parents of node $i$ that are afflicted. \\
$\pi(i)$ & Probability of affliction by an intra-neighbor of node $i$. \\
$\pi^{\prime}(i)$ & Probability of affliction by an inter-parent of node $i$. \\
$T$ & Time horizon (or number of time-steps) considered for experiments. \\
$\Phi$ & Percentage of nodes in the M-IDN selected to be initial spreaders. \\
\hline$C$ Certain notation (such as $\left.N_{A}\right)$ applies to both networks, respectively with the network parameter designated
\end{tabular}


former representing directed edges from layer $A$ to layer $B$ and the latter representing the opposite.

We consider two nodes belonging to the same network with edges between each other as intra-neighbors. Additionally, we consider a node $i$ with a directed edge to a node $j$ that belongs to a different network as an inter-parent. Formally, the set of intra-neighbors for node $i$ in some layer $A$ can be defined as $U_{A}^{\text {intra }}(i)=\left\{j \in A \mid(i, j) \in E_{A}\right\}$, with $\left|U_{A}^{\text {intra }}(i)\right|$ representing the intra-degree of node $i \in A$. Further, we formally define the set of inter-parents belonging to some layer $B$ of a node $i$ belonging to some layer $A$ as $U_{A}^{\text {inter }}(i)=\left\{j \in B \mid M_{B A}(j, i)=1\right\}$.

Under this model, a node has a probability that it will become afflicted only under the case that the proportion of its afflicted neighbors reach or exceed the designated threshold. This model relies on specified a $p_{\max }$ value for each set of connectivity between networks - for our work, we consider $p_{\max (A A)}, p_{\max (A B)}, p_{\max (B B)}$, and $p_{\max (B A)}$. This is a general parametric model that incorporates many previous models as special cases. For the two networks composing the M-IDN topology for our study, we introduce two different threshold functions: $k_{a a}(i) \in(0,1]$ for $i \in A$, and $k_{b b}(i) \in(0,1]$ for $i \in B$ to model the propagation across the nodes of the same network. We also introduce two other threshold functions: $k_{a b}(i) \in(0,1]$ for $i \in B$, and $k_{b a}(i) \in(0,1]$ for $i \in A$, to model propagation across the two interdependent networks, from layer $A$ to layer $B$ and vice versa, respectively. We denote $\pi(i)$ to be the probability of phenomena propagation to afflict node $i$ due to the propagation of intra-neighbors of $i$, and $\pi^{\prime}(i)$ to be the probability of phenomena propagation to afflict node $i$ due to the propagation of inter-parents of $i$.

Assuming that a fraction $\alpha(i)$ of intra-neighbors of node $i$ are already afflicted, the probability that phenomena propagates to node $i$ within one time-step is defined in Eq. 1,

$$
\pi(i)=\left\{\begin{array}{cl}
\alpha(i) \cdot p_{\max (A A)}(i) & \text { if } \alpha(i) \geq k_{a a}(i) \\
0 & \text { if } \alpha(i)<k_{a a}(i)
\end{array}\right.
$$

where $p_{\max (A A)}(i)$ represents the probability that node $i$ is afflicted within one time step, when all of its intra-neighbors are already afflicted. Similarly, node $i$ may become afflicted due to the propagation of some of its inter-parents. Let $\beta(i)$ be the fraction of inter-parents of a node $i$ that are already afflicted. Thus, the probability that phenomena propagates from inter-parents of node $i$ to node $i$ within one time step is calculated as defined in Eq. 2,

$$
\pi^{\prime}(i)=\left\{\begin{array}{cl}
\beta(i) \cdot p_{\max (A B)}(i) & \text { if } \beta(i) \geq k_{b a}(i) \\
0 & \text { if } \beta(i)<k_{b a}(i)
\end{array}\right.
$$

where $p_{\max (B A)}$ represents the probability that node $i$ in layer $A$ is afflicted within one time step, when all of its inter-parents in $B$ are already afflicted. We use a similar notation for node $j \in B$ by defining equations analogous to Eqs. 1 and (2), where we use the thresholds $k_{B B}$ and $k_{A B}$ to express the required fraction of intra-neighbors and inter-parents of node $j$ that must be afflicted before $j$ can become afflicted with positive probability. Also, the probability that node $j$ in layer $B$ is afflicted within one time step, when all of its inter-parents in $A$ are already afflicted will be shown by $p_{\max (A B)}$. Note that $p_{\max (A B)}$ and $p_{\max (B A)}$ are not necessarily equal. 


\section{Generalization for many layers}

This model can easily be extended to consider M-IDN topologies consisting of some abstract $m$ layers. In this work, we only consider M-IDN topologies with two layers, so we provide notation to understand the formal definitions of this model with respect to that (e.g., $M_{A A}, p_{\max (A B)}$, etc.). However, this model can also be used to consider MIDN topologies made of more than two layers. For instance, in an M-IDN topology of three layers $(A, B$, and $C$ ), you would consider all the same formal definitions, but include parameter permutations that involve $C-$ such as $M_{C C}, M_{C A}, M_{A C}, M_{C B}, M_{B C}$, etc.

The decision to solely focus on M-IDN topologies of two layers for this work was motivated by the curiosity to explore how varying interconnectivity, selection strategies, sizes, and other parameters affect the evolution over time of the phenomena propagation process. More specifically, we would like to study to what extent interdependency between networks and the knowledge of network topology can help the speed of propagation in a M-IDN network.

\section{Phenomena propagation process}

The temporal evolution of phenomena propagation is modeled as a Markov model. This is because the next state of the network will only depend on the current state of the network and it is independent of how the network reaches its current state. In the following, we describe the elements of our proposed Markov model.

\section{State definition}

We denote by $S_{T}$ the set of all possible states of the model, where each state is defined as a vector $s=(\overbrace{s_{1}, s_{2}, \ldots, s_{N_{A}}}^{A}, \overbrace{s_{N_{A}+1}, s_{N_{A}+2}, \ldots, s_{N_{A}+N_{B}}}^{B})$, where $s_{i}=1$ for $i \leq N_{A}$ if node $i \in A$ is afflicted, and $s_{i}=0$ if it is not afflicted. Similarly, for $i>N_{A}, s_{i}=1$ is one if node $i-N_{A}$ of layer $B$ is afflicted, and $s_{i}=0$ otherwise. Therefore, the initial state of the phenomena propagation process is $S_{0}=\left(s_{1}, s_{2}, \ldots, s_{N_{A}}, s_{N_{A}+1}, s_{N_{A}+2}, \ldots, s_{N_{A}+N_{B}}\right)$, where $s_{k}=1$ if $k \leq N_{A} \wedge k \in A \cap F_{0}^{A}$ or $k>N_{A} \wedge k \in B \cap F_{0}^{B}$, while $s_{k}=0$ otherwise. $F_{0}^{A}$ and $F_{0}^{B}$ are the set of initial spreaders in layers $A$ and $B$, respectively.

According to our proposed model, a node can become afflicted only if the proportion of its afflicted intra-neighbors and/or inter-parents exceed a given threshold. Therefore, not all the binary vectors of $N_{A}+N_{B}$ elements represent a feasible state of the process. Note that a node of layer $A$ may become afflicted due to the consequences of the initial spreaders in layer $A$, layer $B$, or both. The goal of this paper is to analyze the impact of concurrent phenomena propagation in M-IDNs to gain a better understanding of the most robust interdependent network topology. Our propagation model and the Markov model provide a general framework that allows for further investigation of different types of phenomena propagation/impact.

\section{Transition probabilities}

Based on our previous definitions, we calculate the one-step transition probability matrix of the process $\wp \in\{0,1\}^{\left|S_{T}\right| \times\left|S_{T}\right|}$, whose generic element $\left.\wp\right|_{s, s^{\prime}}$ gives the probability that the network transits from state $s$ to state $s^{\prime}$. We let $\Delta s=s^{\prime}-s$. The $j$-th element of vectors $\Delta s$ and $s$ are represented by $\Delta s_{j}$ and $s_{j}$, respectively. In order to calculate $\left.\wp\right|_{s, s^{\prime}}$, we need to identify two types of transitions: $i$ ) transitions where $\Delta s_{j}=0$, and $i$ ) transitions where 
$\Delta s_{j}=1$. We denote an indicator function $I$ (cond) where cond is a boolean value and $I($ true $)=1, I($ false $)=0$. A formal definition for $\left.\wp\right|_{s, s^{\prime}}$ is provided in Eq. 3,

$$
\left.\wp\right|_{s, s^{\prime}}=\prod_{j=1}^{N_{A}+N_{B}}\left[f(j) I\left(\Delta s_{j}=0\right)+f^{\prime}(j) I\left(\Delta s_{j}=1\right)\right],
$$

where $f(j)$ denotes the probability that there is no change in the $j$-th component of the network state when transitioning from state $s$ to state $s^{\prime}$, and $f^{\prime}(j)$ is the probability that there is a change of status for the $j$-th node of the network, going from a working node to a afflicted node. It is important to note that $\Delta s_{j}=0$ happens in two cases: $i$ ) the related node is already afflicted, as we do not consider recovery or restoration of the network in this paper, and $i$ ) the related node is currently working and it will remain the same as the proportion of afflicted neighbors/parents does not meet the threshold or, although the propagation threshold is met, the propagation did not occur in the current time-step (referring to the probabilistic nature of the propagation model).

We also calculate the value of $f(j)$ and $f^{\prime}(j)$ by separating the terms related to layers $A$ and $B$. In particular, the probability of having a change in the $j$-th element of the interdependent network when the network state changes from $s$ to $s^{\prime}$ can be calculated in the definition for Eq. 4.

$$
f(j)=\left\{\begin{array}{cl}
g_{a}(j) & \text { if } j \leq N_{A} \wedge s_{j}=0 \\
g_{b}(j) & \text { if } j>N_{A} \wedge s_{j}=0 \\
1 & \text { if } \quad s_{j}=1
\end{array}\right.
$$

Then, $g_{a}(j)$ can be calculated as defined in Eq. 5,

$$
\begin{aligned}
g_{a}(j)= & I\left(\alpha(j)<k_{a a}(j)\right) \cdot I\left(\beta(j)<k_{b a}(j)\right)+ \\
& I\left(\alpha(j) \geq k_{a a}(j)\right) \cdot I\left(\beta(j)<k_{b a}(j)\right) \cdot \bar{\pi}(j)+ \\
& I\left(\alpha(j)<k_{a a}(j)\right) \cdot I\left(\beta(j) \geq k_{b a}(j)\right) \cdot \bar{\pi}^{\prime}(j)+ \\
& I\left(\alpha(j) \geq k_{a a}(j)\right) \cdot I\left(\beta(j) \geq k_{b a}(j)\right) \cdot \bar{\pi}^{\prime}(j) \cdot \bar{\pi}(j)
\end{aligned}
$$

where $\bar{\pi}(j)=1-\pi(j)$ and $\bar{\pi}^{\prime}(j)=1-\pi^{\prime}(j)$, with $\pi(j)$ and $\pi^{\prime}(j)$ defined in Eqs. 1 and 2, respectively.

The term $g_{b}(j)$, will be similarly calculated for the nodes located in layer $B$, by replacing $k_{a a}$ with $k_{b b}$, and $k_{b a}$ with $k_{a b}$ in Eq. 5 . Note that according to our definition, the nodes of the M-IDN system are enumerated from 1 to $N_{A}+N_{B}$, therefore, $k_{a a}$ and $k_{b a}$ are only defined for $j \leq N_{A}$ and $k_{b b}, k_{a b}$ are only defined for $N_{A}<j \leq N_{A}+N_{B}$.

The term $f^{\prime}(j)$ denotes the probability that there is a change in the $j$-th component of the state vector, when the network is transitioning from state $s$ to state $s^{\prime}$. We split $f^{\prime}(j)$ in the contributions related to the two layers $A$ and $B$. Therefore,

$$
f^{\prime}(j)=\left\{\begin{array}{c}
g_{a}^{\prime}(j) \text { if } j \leq N_{A} \wedge s_{j}=0 \\
g_{b}^{\prime}(j) \text { if } j>N_{A} \wedge s_{j}=0 \\
0 \text { if } \quad s_{j}=1
\end{array}\right.
$$

Similar to our previous note, we can calculate the term $g_{a}^{\prime}(j)$ as follows,

$$
\begin{aligned}
g_{a}^{\prime}(j)= & I\left(\alpha(j) \geq k_{a a}(j)\right) \cdot I\left(\beta(j) \geq k_{b a}(j)\right) . \\
& \left(\pi(j) \pi^{\prime}(j)+\bar{\pi}(j) \pi^{\prime}(j)+\pi(j) \bar{\pi}^{\prime}(j)\right)+ \\
& I\left(\alpha(j)<k_{a a}(j)\right) \cdot I\left(\beta(j) \geq k_{b a}(j)\right) \cdot \pi^{\prime}(j)+ \\
& I\left(\alpha(j) \geq k_{a a}(j)\right) \cdot I\left(\beta(j)<k_{b a}(j)\right) \cdot \pi(j)
\end{aligned}
$$


Using a similar argument, we can write similar equations for the nodes in layer $B$, i.e. $g_{b}^{\prime}(j)$ can be calculated.

Note: Based on the definition of transition probabilities, we observe that the speed of phenomena propagation will increase, if and only if $f^{\prime}(j) \gg f(j)$ in Eq. 3. This is because, under this condition, the second term in Eq. 3 will dominate the transition probability, meaning that the probability that $\Delta s_{j}=1$ is much larger than that of $\Delta s_{j}=0$, therefore the number of afflicted nodes increases faster over time. Looking at $f(j)$ and $f^{\prime}(j)$, we can see that both are functions of $p_{\max (* *)}$. By increasing the value of $p_{\max (* *)}$, we will have $f^{\prime}(j)>f(j)$, as $f^{\prime}(j)$ is an increasing function of $\pi$ and $\pi^{\prime}$, and thus an increasing function of $p_{\max (* *)}$. On the other hand, $f(j)$ is a decreasing function of these probabilities.

\section{Expected absorption time}

Phenomena propagation in an M-IDN system can be seen as an absorbing Markov chain, where the absorbing states are defined as one of the following: $i$ ) all nodes of the M-IDN system are afflicted, and $i$ ) no working node can meet the phenomena propagation condition. The set of absorbing states depends on the sets of initial spreaders $\left(F_{0}^{A}\right.$ and $\left.F_{0}^{B}\right)$ and the propagation thresholds. Therefore, we may get different absorbing states for the same network topology when the set of initial spreaders in $A$ and $B$ are different. Given that we have the states of the network and transition probabilities, we can use standard techniques, used in (Charles et al. 1997), for analyzing our proposed Markov process and to calculate the expected absorption time. However, the state space for a reasonably sized network would be very large as we are considering the state of a network's individual elements. Due to this limitation, we have only tested our model using networks of a relatively small size for the proposed Markov model. We then use this to validate the correctness of our simulations that have been built to handle networks of larger sizes while using the same phenomena propagation model. An example of simulation validation can be found in (Khamfroush et al. 2016). As the focus of this work is to use extensive simulations to provide useful guidelines on the vulnerability of different network topologies, we focus on simulation setup and results in the following sections.

\section{Description of experiments}

We investigate how different initial spreader selection strategies affect phenomena propagation in M-IDN systems. To study this, we perform and analyze extensive simulations. To be thorough, we consider the time evolution of phenomena propagation of M-IDN systems with varying network topologies, coupling strategies, inter-edge density, average degree, and the choice of initial spreaders. For this work, we are particularly interested in how single-layer initial spread in Scenarios 1-1 and 2-1 compares to multi-layer initial spread in Scenarios 1-2 and 2-2.

\section{Tested scenarios}

For this work, we consider an M-IDN topology composed of two layers $A$ and $B$. For our simulations, we considered four possible scenarios for initial spreader selections. In each scenario considered, we designate $\left|F_{0}\right|=\left\lfloor\Phi \cdot\left(N_{A}+N_{B}\right)\right\rfloor$ where $\Phi$ is the percentage of nodes we select to be initial spreaders. These scenarios incorporate what we call "single- 
layer selection" or "two-layer selection". If a scenario uses the former, then layer $A$ will have $\left|F_{0}\right|$ nodes selected as initial spreaders; if a scenario uses the latter, then layers $A$ and $B$ will both have $\left|F_{0}\right| / 2$ nodes selected as initial spreaders. Below are the scenarios considered:

- Scenario 1-1: Single-layer selection, initial spreaders chosen at random.

- Scenario 1-2: Two-layer selection, initial spreaders chosen at random.

- Scenario 2-1: Single-layer selection, initial spreaders chosen by high degree centrality.

- Scenario 2-2: Two-layer selection, initial spreaders chosen by high degree centrality.

\section{Interconnectivity models}

For our simulations, we consider a variety of interconnectivity for thorough examination of our core question. With this in mind, we consider the notion of inter-connections (represented by directed inter-edges) being established at random or by design. Additionally, we also aim to explore how low or high numbers of inter-connections affect phenomena propagation.

There are a lot of considerations in our interconnectivity model. First, we introduce the four interconnectivity cases considered in our experiments:

- Sparse \& Random: In this model, $8 \%$ of nodes are selected to have inter-edges directed to nodes in the other network. $4 \%$ of nodes in layer $A$ (chosen at random) have inter-edges to nodes in layer $B$ (chosen at random), and vice versa.

- Dense \& Random: In this model, 20\% of nodes are selected to have inter-edges directed to nodes in the other network. 10\% of nodes in layer $A$ (chosen at random) have inter-edges to nodes in layer $B$ (chosen at random), and vice versa.

- Sparse \& Designed: In this model, 8\% of nodes are selected to have inter-edges directed to nodes in the other network. $4 \%$ of nodes in layer $A$ (based on degree centrality) have inter-edges to nodes in layer $B$ (based on degree centrality), and vice versa.

- Dense \& Designed: In this model, 20\% of nodes are selected to have inter-edges directed to nodes in the other network. $10 \%$ of nodes in layer $A$ (based on degree centrality) have inter-edges to nodes in layer $B$ (based on degree centrality), and vice versa.

Additionally, in the case of designed interconnectivity (Sparse-Designed or DenseDesigned), we consider how nodes are chosen to have inter-edges. We are interested in exploring how nodes of high degree and low degree affect the evolution of phenomena propagation. For this, we consider three cases of how designed selection of nodes with inter-edges takes place:

- Max-Max: Nodes of highest degree in the currently considered network are designed to have inter-edges to nodes of the highest degree in the other network, and vice versa.

- Max-Min: Nodes of highest degree in layer $A$ are designed to have inter-edges to nodes of the lowest degree in layer $B$ and nodes of lowest degree in layer $B$ have inter-edges to nodes of highest degree in layer $A$.

- Min-Min: Nodes of lowest degree in the currently considered network are designed to have inter-edges to nodes of the lowest degree in the other network, and vice versa. 


\section{Tested network topologies}

\section{Synthetic topologies}

For synthetic topologies, we use three well-studied random generative models for network topologies: Erdös-Rényi (ER) model (Erdos and Rényi 1960), Barabási-Albert (BA) model (Barabási and Albert 1999), and the Watts-Strogatz (WS) model (Watts and Strogatz 1998). The ER model produces what are commonly referred to as purely random graphs; the BA model produces topologies that maintain a power law degree distribution; and the WS model produces topologies with the small-world property.

To be thorough, we consider all permutations of the random generative models for a two-layer M-IDN topology. For instance, an M-IDN topology composed of two layers $A$ and $B$ that are both constructed by the ER model will be referred to as an ER-ER topology (coinciding with $A-B$ ). It is important to note that network topologies are not transitive in our experiments - i.e., an ER-SW topology is not equivalent to an SW-ER topology. The reason for this is that a single-layer selection scenario only selects initial spreaders in layer $A$, thus a simulation using an ER-SW topology is not comparable to a simulation using an SW-ER topology. To be clear, in all of our experiments, we generate a synthetic, random M-IDN topology in each Monte-Carlo run.

\section{Real-world topologies}

For comparison, we also run simulations using real-world multiplex topologies. These topologies come from different domains - social networks, genetic networks, etc. A key difference between these topologies and our synthetic topologies is the inter-connection between layers $A$ and $B$. In our synthetic topologies, we consider Sparse/Dense and Designed/Random inter-connections. In our real-world topologies, each node exists across all layers with inter-edges to each other node corresponding to it across all layers. For a concise description o fhte real-world topologies considered for this work, refer to Table 2 .

\section{Simulation setup}

Here, we review some of the constants that are maintained across all simulations considered for our experiments and review some other experimental choices that have not been addressed thus far. For any simulation setup, we consider the evolution of phenomena propagation over a time horizon of $T=200$ time steps. In the plots presented for our results, we let the $\mathrm{x}$-axis represent the time steps comprising the time horizon $T$; while the $y$-axis represents the number (or percentage) of afflicted nodes in the entire M-IDN topology.

Table 2 Overview of the real-world multiplex network topologies considered for simulations in this work

\begin{tabular}{llll}
\hline Title & $|V|$ & $|E|$ & $\langle k\rangle$ \\
\hline FAO Multiplex Trade Network (De Domenico et al. 2015) & 214 & 31,8346 & 37.6 \\
CKM Physician Innovation Social Network (Coleman et al. 1957) & 246 & 1551 & 4.74 \\
Human HIV Multiplex Genetic Network (Stark et al. 2006) & 1005 & 1355 & 2.21 \\
Arabidopsis Multiplex GPI Network (Stark et al. 2006) & 6980 & 18,654 & 3.45 \\
\hline
\end{tabular}

These network topologies all include more than two layers. For this work, we simply consider the two layers with the most edges. Note: Since these are multiplex networks and each node is in each layer, $\left|V_{A}\right|=\left|V_{B}\right|=|V|$ 
Additionally, for our synthetic networks, we consider cases where $N_{A}=N_{B}=100$ and $N_{A}=N_{B}=500$. For all synthetic topologies, we consider an average degree $\langle k\rangle=4$. We evaluated how average degree affects phenomena propagation before making this decision. For our considered threshold parameter values, we observe that higher average degree corresponds with significantly less affliction throughout the phenomena propagation process - refer to Fig. 1.

Additionally, we are interested in the cases where values for our probabilistic thresholdbased model are $i$ ) the same for layer $A$ and $B$ and $i i$ ) where they are different. The former case is what we refer to as homogeneous propagation while the latter case is referred to as heterogeneous propagation. For heterogeneous propagation, we use the following threshold values: $k_{a a}=0.5, k_{b b}=0.2, k_{a b}=0.3$, and $k_{b a}=0.8$. For homogeneous propagation, we use the following threshold values: $k_{a a}=k_{b b}=0.3$ and $k_{a b}=k_{b a}=0.5$. These threshold values are used for all simulations, both synthetic and real-world.

\section{Results}

To reiterate, we consider a variety of parameters for a simulation. To cope for randomness, each simulation setup is run a number of $M=100$ Monte Carlo runs, with results being averaged across these runs. Due to the sheer number of parameters considered, our results span hundreds of simulation setups. Therefore, we want to highlight important pieces of data analysis in hopes that key conclusions can be made. So, we elect to show plots of some of the more interesting simulation results and will describe general trends

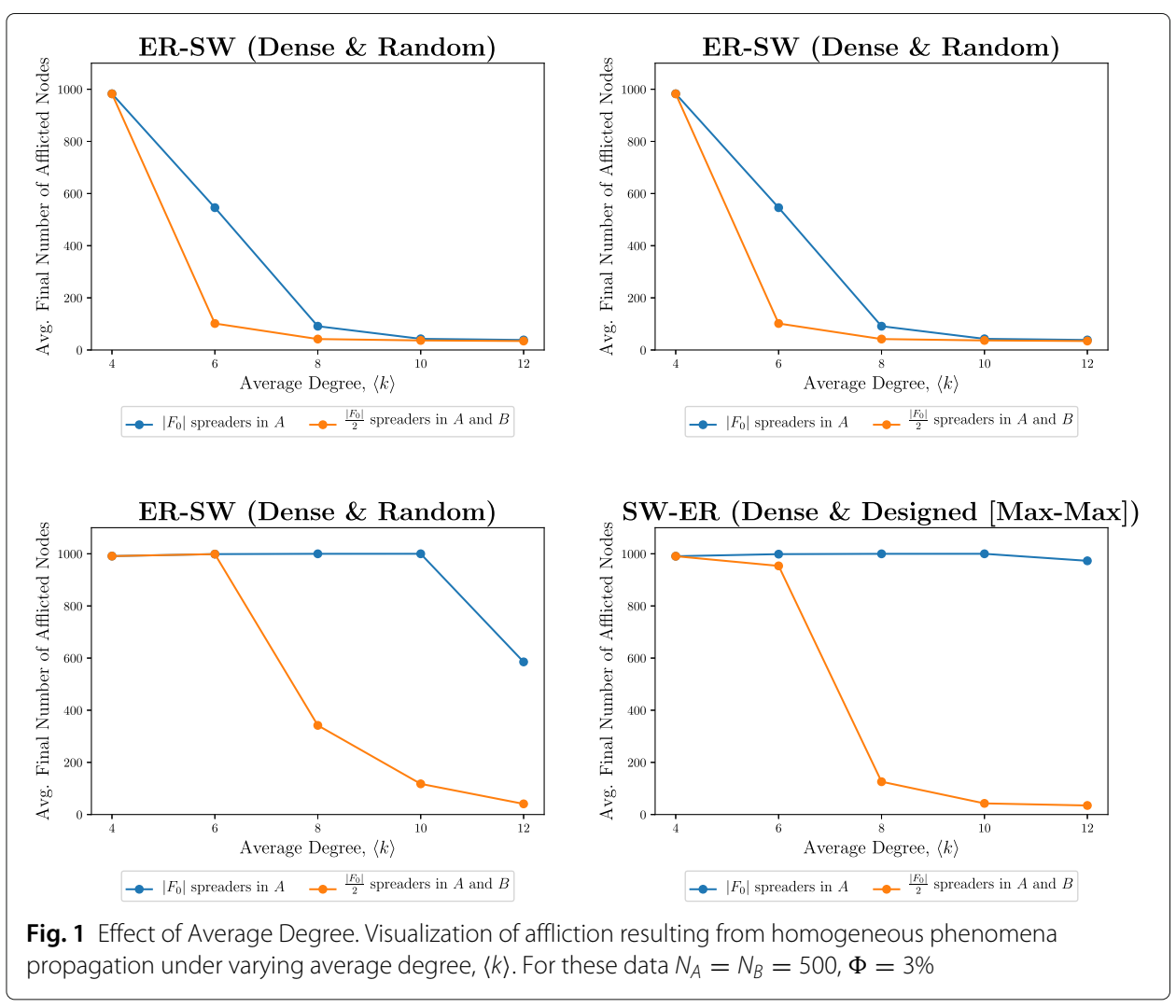


throughout. For brevity, we say "single-layer selection" to refer to Scenarios 1-1 and 2-1 and "two-layer selection" to refer to Scenarios 1-2 and 2-2.

Interestingly, for the interconnectivity cases we consider for this work, a noteworthy trend across most synthetic simulation setups is that two-layer selection of initial spreaders appears generally to be more effective than single-layer selection in affecting the entire M-IDN topology. In many cases, this selection strategy afflicts the entire M-IDN topology early in the propagation process; whereas the propagation under single-layer selection commonly saturates early in the propagation process without afflicting the entire topology (or full affliction is attained after two-layer selection). As an additional observation, we observed that as we experimentally increased average degree $\langle k\rangle$, single-layer selection outperforms two-layer selection - this was observed before designating $\langle k\rangle=4$ but we felt worth mentioning. We also note that as $\Phi$ increases, generally two-layer selection outperforms single-layer seleciton.

\section{Homogeneous simulations}

Here we investigate the results of our homogeneous simulations. As a reminder, we refer to homogeneous simulations as simulations where the threshold values for our propagation model are constant across both layers $A$ and $B$ in the layers of our M-IDN topology.

Intuitively, Sparse interconnectivity essentially dampens the possible effects of phenomena propagation under our model. Our results reflect this intuition. Refer to Fig. 2 for a sample of these simulation results. For a detailed overview of affliction w.r.t. to time evolution, refer to Table 3. Overall, we see in the homogeneous case, that Scenario 2-2 (two-layer selection by degree centrality) is the most effective (w.r.t. to time and percentage of affliction) at reaching near full affliction across most interconnectivity cases considered.

\section{Designed max-max}

We observe for homogeneous simulations with Dense interconnectivity that the variance across Monte-Carlo runs is incredibly small across all considered topologies, network sizes, and $\Phi$. This observation makes sense upon further consideration. Consider the BA random model for network topologies. Due to preferential attachment, nodes are more likely to coalesce and associate with nodes of high degree (forming "hubs") - which are selected to exhibit interconnection under Scenarios 2-1 and 2-2. If the central, highly

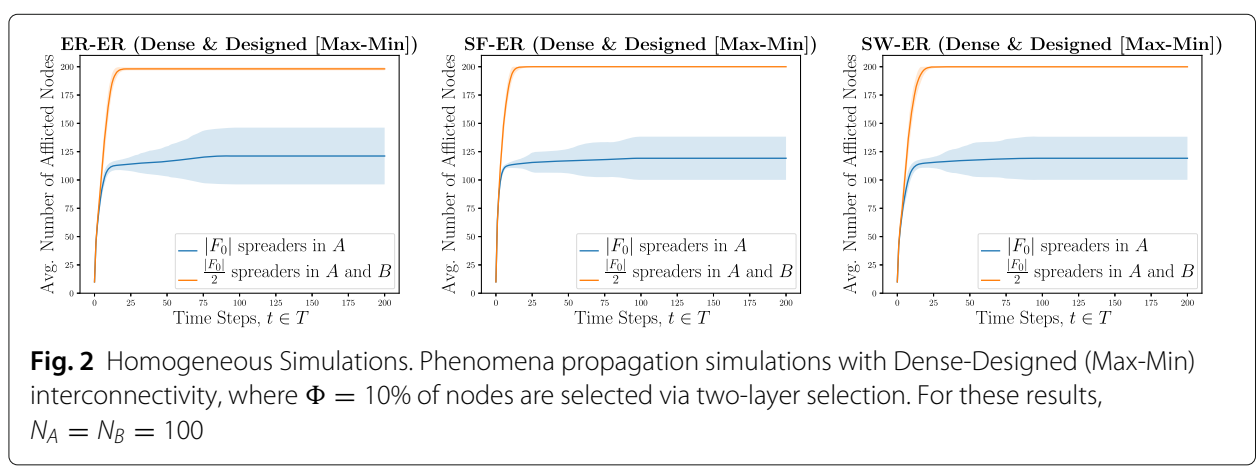


Table 3 Homogeneous data for when $N_{A}=N_{B}=500$ and $\Phi=3 \%$

\begin{tabular}{|c|c|c|c|c|c|c|c|c|c|}
\hline \multirow[b]{2}{*}{ Scen. } & \multirow[b]{2}{*}{ Interconnectivity } & \multicolumn{4}{|c|}{ Single-Layer Selection } & \multicolumn{4}{|c|}{ Multi-Layer Selection } \\
\hline & & $t=50$ & $t=100$ & $t=150$ & $t=200$ & $t=50$ & $t=100$ & $t=150$ & $t=200$ \\
\hline \multirow[t]{8}{*}{1} & Dense (Max-Max) & 806 & 934 & 942 & 942 & 638 & 853 & 874 & 876 \\
\hline & Dense (Max-Min) & 492 & 740 & 789 & 798 & 602 & 874 & 915 & 919 \\
\hline & Dense (Min-Min) & 503 & 725 & 785 & 794 & 298 & 615 & 720 & 741 \\
\hline & Dense (Random) & 738 & 962 & 971 & 971 & 545 & 864 & 907 & 912 \\
\hline & Sparse (Max-Max) & 645 & 906 & 928 & 929 & 323 & 659 & 717 & 724 \\
\hline & Sparse (Max-Min) & 438 & 501 & 511 & 513 & 341 & 621 & 694 & 715 \\
\hline & Sparse (Min-Min) & 440 & 497 & 504 & 505 & 205 & 415 & 491 & 513 \\
\hline & Sparse (Random) & 484 & 680 & 761 & 776 & 259 & 592 & 688 & 706 \\
\hline \multirow[t]{8}{*}{2} & Dense (Max-Max) & 994 & 994 & 994 & 994 & 994 & 994 & 994 & 994 \\
\hline & Dense (Max-Min) & 723 & 840 & 846 & 846 & 997 & 997 & 997 & 997 \\
\hline & Dense (Min-Min) & 666 & 818 & 829 & 830 & 998 & 998 & 998 & 998 \\
\hline & Dense (Random) & 986 & 994 & 994 & 994 & 994 & 994 & 994 & 994 \\
\hline & Sparse (Max-Max) & 993 & 993 & 993 & 993 & 992 & 993 & 993 & 993 \\
\hline & Sparse (Max-Min) & 531 & 533 & 537 & 538 & 996 & 997 & 997 & 997 \\
\hline & Sparse (Min-Min) & 525 & 527 & 530 & 531 & 997 & 998 & 998 & 998 \\
\hline & Sparse (Random) & 626 & 780 & 812 & 816 & 994 & 994 & 994 & 994 \\
\hline
\end{tabular}

Each value corresponds to the average number of afflicted nodes at the respective time-step, $t$, across all considered Monte-Carlo runs for all synthetic topologies

connected node of this hub is afflicted, its lesser connected neighbors are more vulnerable to affliction because that high degree node likely makes up a sizable proportion of its neighbors (thus having more impact on its threshold to affliction). For homogeneous simulations with Sparse interconnectivity, we also see very little variance - though not as small.

For Dense interconnections, generally the single-layer selection of initial spreaders outperforms the two-layer selection strategy. The difference between these two strategies in this setup is not particularly notable, but the general trend is that single-layer selection is slightly more effective. Meanwhile, for Sparse interconnections, the general trend holds. However, the difference between single-layer and two-layer selection strategy is more prominent. Additionally, it is important to note that as $\Phi$ increases, the two-layer selection strategy begins to outperform the single-layer selection strategy. This implies that if you have more budget to select more initial spreaders under these parameters, twolayer selection would be most effective. However, if you have a more conservative budget, single-layer selection would be more appropriate.

\section{Designed max-min}

For both Dense and Sparse interconnectivity, we observe that our averaged data shows that two-layer selection notably outperforms single-layer selection in all cases. Under Dense interconnectivity, the variance of the resulting phenomena propagation for singlelayer selection is notably large; while the variance of the resulting phenomena propagation for two-layer selection in this case is very small. However, under Sparse interconnectivity, the variance of the resulting phenomena propagation for single-layer selection is significantly smaller than under Dense interconnectivity.

It is important to note, that under this interconnectivity, two-layer selection results in full-affliction in nearly every case. Single-layer selection is not as effective, with no 
simulation using single-layer selection having comparable affliction rates to two-layer selection. The performance of single-layer selection is starkly less impressive under Sparse interconnectivity - with propagation saturating early on with about $50 \%$ affliction across all cases. This is likely due to initial spreaders exclusively selected in layer $A$ propagate affliction to nodes minimum degree nodes in layer $B$. As a result, layer $A$ is roughly $90 \%$ afflicted whereas layer $B$ roughly $10 \%$ afflicted.

\section{Designed min-min}

With an increase in the size of the networks, single-layer selection is notably more effective than in smaller network sizes - reaching full affliction (on average) in some cases. Otherwise, results for this set of experiments is very comparable to Designed Max-Min. However, there does appear to be less variance for single-layer selection. In the Max-Min case, some nodes in the layer $B$ can cross-propagates though maximum degree nodes under two-layer selection. However, in the case of Min-Min, it this does not happen. This is likely why the difference is not as initially prominent.

\section{Random}

There is a stark difference between Dense interconnectivity and Sparse interconnectivity in this case. In Dense interconnectivity, the performance between single-layer selection and two-layer selection is marginal - though two-layer selection has a slight edge. Both selection strategies, on average, result in full affliction (or very close to it) in all cases considered - when $N_{A}=N_{B}=500$ all experiments reached full affliction. Additionally, variance of performance was very low under Dense interconnectivity. It should be noted that when $\Phi$ is a low value (e.g., $\Phi=3 \%$ ), there is a split in certain topologies where singlelayer selection performs better in terms of overall affliction. However, as $\Phi$ increases, two-layer selection begins to close the gap in these topologies and eventually overtakes it in terms of affliction w.r.t. to time.

These remarks cannot be made for this experiment under Sparse interconnectivity. When $N_{A}=N_{B}=100$, the performance of single-layer selection would typically hover in the range of about $50 \%-70 \%$ affliction.

\section{Heterogeneous simulations}

Here we investigate the results of our heterogeneous simulations. As a reminder, we refer to heterogeneous simulations as simulations where the threshold values for our propagation model are unique across both layers $A$ and $B$ in the layers of our M-IDN topology. There are no standout trends across the different models of interconnectivity. Below is a brief summary of the general behavior of these simulations. Generally, for the Heterogeneous case (under the threshold values we use), the most apparent trend is that single-layer selection seems to be a more potent selection strategy overall than two-layer selection. It is important to note that this trend is not guaranteed to hold for different threshold values under this propagation model.

For this work, under single-layer selection, initial spreader selection takes place in layer $A$, which is more robust to affliction by inter-parents and intra-parents. Since the selection is concentrated in the more robust layer, the results show that we get more affliction in that layer. Subsequently, the cross-propagation is not as difficult since the layer $A$ is more vulnerable. As for two-layer selection, less affliction occurs in layer $A$ 
due to its robustness since half of the resources for selection are used on the vulnerable layer. For a more detailed overview of affliction w.r.t. to time evolution, refer to Table 4.

For both Sparse and Dense interconnectivity, when $N_{A}=N_{B}=100$, the general trend is that two-layer selection outperforms single-layer selection when the percentage of nodes selected to be initial spreaders is smaller. Once $10 \%$ of the nodes are selected to be initial spreaders, for both cases, the single-layer selection strategy begins to overtake the twolayer selection strategy. However, the difference in performance is marginal and not highly significant.

However, when $N_{A}=N_{B}=500$, under Dense interconnectivity, the strategies perform nearly identically until $10 \%$ of nodes are selected as initial spreaders. In which case, singlelayer selection begins to have the advantage. The advantage in this case, is notable and is quite significant when compared to the experiments where $N_{A}=N_{B}=100$. Also, the variance in the resulting phenomena propagation is very high when the percentage of nodes selected to be initial spreaders is small.

\section{Real-world simulations}

Results for real-world simulations depart from the general trend we observe in our synthetic simulations. In our real-world simulations, under designed initial spreader selection (Scenario 2), we observe that single-layer initial spreader generally outperforms multi-layer initial spreader selection. However, the important detail to note is that the difference is not as notable as with our synthetic simulations. Additionally, the propagation process converges in a few time steps over the entire time horizon $T$. The amount of affliction that occurs varies based on value of $\Phi$. In Scenario 2-2, typically the affliction struggles to afflict additional nodes beyond the set of initial spreaders. However, in the other Scenarios considered, we see that affliction typically saturates at around $2 \cdot\left|F_{0}\right|$. The

Table 4 Heterogeneous data for when $N_{A}=N_{B}=500$ and $\Phi=3 \%$

\begin{tabular}{|c|c|c|c|c|c|c|c|c|c|}
\hline \multirow[b]{2}{*}{ Scen. } & \multirow[b]{2}{*}{ Interconnectivity } & \multicolumn{4}{|c|}{ Single-Layer Selection } & \multicolumn{4}{|c|}{ Multi-Layer Selection } \\
\hline & & $t=50$ & $t=100$ & $t=150$ & $t=200$ & $t=50$ & $t=100$ & $t=150$ & $t=200$ \\
\hline \multirow[t]{8}{*}{1} & Dense (Max-Max) & 821 & 902 & 913 & 917 & 834 & 891 & 899 & 901 \\
\hline & Dense (Max-Min) & 539 & 711 & 737 & 743 & 817 & 862 & 872 & 874 \\
\hline & Dense (Min-Min) & 552 & 586 & 586 & 586 & 571 & 571 & 571 & 571 \\
\hline & Dense (Random) & 573 & 617 & 620 & 620 & 612 & 613 & 613 & 613 \\
\hline & Sparse (Max-Max) & 527 & 546 & 546 & 546 & 708 & 713 & 713 & 713 \\
\hline & Sparse (Max-Min) & 275 & 341 & 347 & 348 & 677 & 691 & 693 & 693 \\
\hline & Sparse (Min-Min) & 350 & 421 & 425 & 425 & 539 & 539 & 539 & 539 \\
\hline & Sparse (Random) & 394 & 434 & 437 & 437 & 554 & 554 & 554 & 554 \\
\hline \multirow[t]{8}{*}{2} & Dense (Max-Max) & 793 & 813 & 823 & 825 & 791 & 812 & 822 & 825 \\
\hline & Dense (Max-Min) & 777 & 793 & 801 & 802 & 779 & 794 & 801 & 802 \\
\hline & Dense (Min-Min) & 740 & 756 & 757 & 757 & 719 & 723 & 723 & 723 \\
\hline & Dense (Random) & 797 & 852 & 869 & 874 & 755 & 755 & 755 & 755 \\
\hline & Sparse (Max-Max) & 715 & 715 & 715 & 715 & 690 & 693 & 693 & 693 \\
\hline & Sparse (Max-Min) & 718 & 718 & 718 & 718 & 674 & 685 & 686 & 686 \\
\hline & Sparse (Min-Min) & 596 & 646 & 648 & 648 & 672 & 691 & 694 & 694 \\
\hline & Sparse (Random) & 685 & 720 & 721 & 721 & 700 & 708 & 708 & 708 \\
\hline
\end{tabular}

Each value corresponds to the average number of afflicted nodes at the respective time-step, $t$, across all considered Monte-Carlo runs for all synthetic topologies 
time evolution of the propagation process for these real-world topologies can be seen in Fig. 3.

The reasoning for this is likely due to the interconnection of the real-world topologies. It is very difficult to find publicly available M-IDN topologies. ComuneLab provides publicly available topological data for multiplex networks, from which we acquired real-world MIDN topological data. However, a key distinction between the real-world topologies we run simulations on from this resource is that they are multiplex networks. Refer to Table 2 for an overview of the real-world multiplex topologies considered for this work.

Multiplex networks are topologies with numerous layers, with each nodes existing across every layer. These multi-layer network models allow for interesting relationships between entities. For instance, a layer in a multiplex network can have edges that represent who retweets who on Twitter, while another layer in the same multiplex network can have edges that represent who replies to who on Twitter. In this example, each node is shared across each layer and inter-edges are only between each node to itself in every other layer. For instance, Donald Trump's Twitter account would be a node shared across all layers in a multiplex Twitter network. The inter-edges this node has will be to the other nodes representing Donald Trump's Twitter account across all other layers. These nodes will have no inter-edges to nodes representing Twitter user accounts other than Donald Trump's.
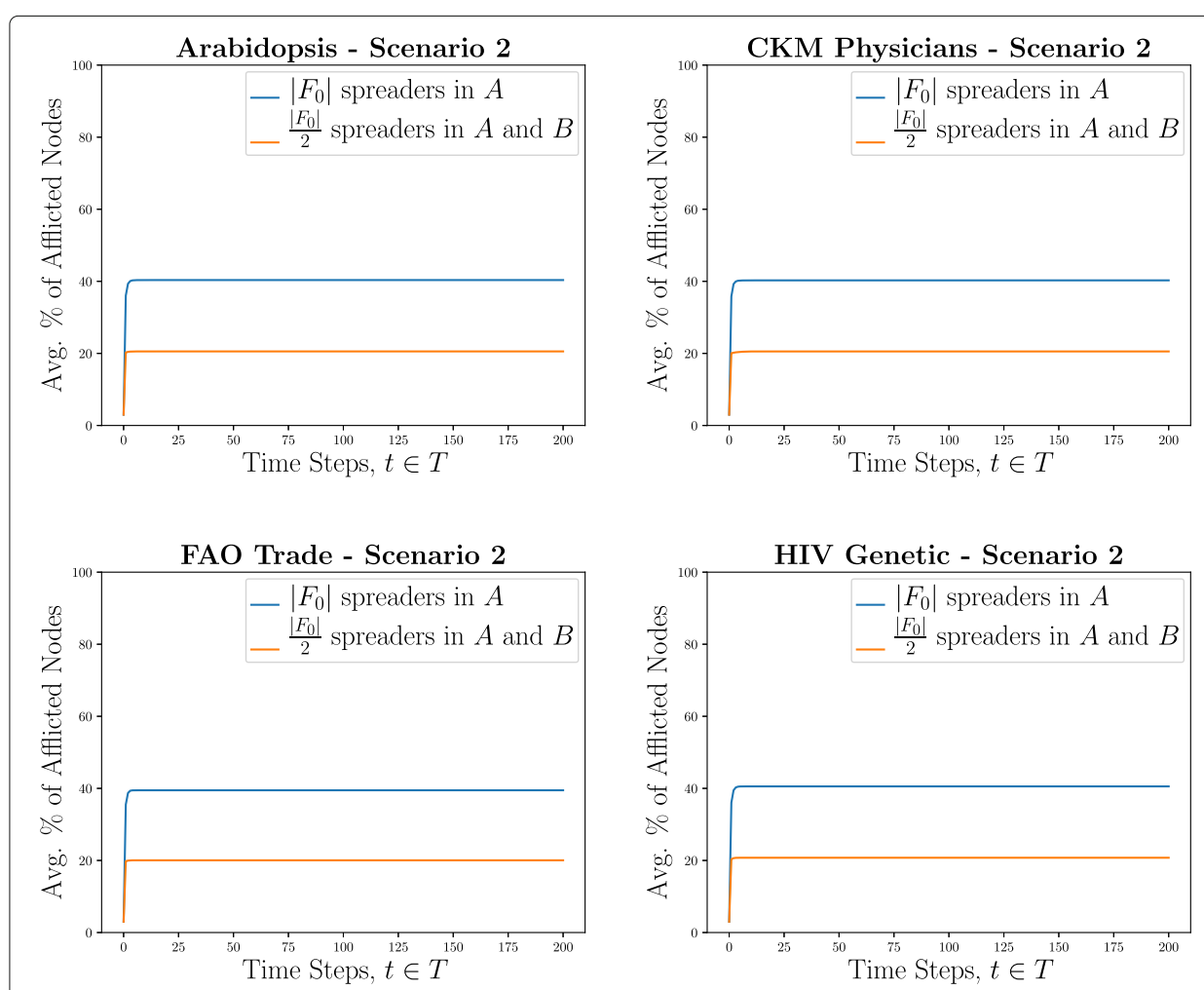

Fig. 3 Real-World Homogeneous Simulation Results. Results are for when $\Phi=20 \%$. Refer to Table 2 for details pertaining to the network topo- logies. Each line represents the percentage of afflicted nodes at the respective time-step, $t$, across all considered Monte-Carlo runs for each real-world topo- logy. We use percentage here due to varying topology sizes of these networks 
This detail is in stark contrast to our synthetic networks. We vary the level of interdependency across our synthetic topology through Sparse/Dense and Random/Designed interconnections. A visualization of what these topologies look like can be found in Fig. 4. Due to this high interconnection between layers, it makes sense that these networks do not exhibit the same behaviors of affliction as our synthetic topologies.

\section{Degree single-layer vs. random multi-layer}

For this work, we are interested in how designed, single-layer selection and random, two-layer selection compare. The interest in this is motivated by the notion that it is theoretically easier to obtain topological structure of one layer within an M-IDN topology. With this knowledge, one could target highly connected nodes in this layer for affliction. With this in mind, we want to compare how a single-layer selection of initial spreaders by degree centrality (Scenario 1-2) compares to a two-layer selection of initial spreaders chosen at random (Scenario 2-1).

From analyzing the results from Tables 4 and 3, we see that single-layer selection by degree centrality (Scenario 2-1) generally outperforms two-layer selection at random (Scenario 1-2) in both the heterogeneous and the homogeneous cases in the vast majority of experiments considered. However, it should be reiterated that single-layer selection selects nodes to be initial spreaders in layer $A$. With this in mind, $k_{a b}=0.3$ (and $k_{b a}=0.8$ ) for heterogeneous cases. The results of this analysis for the heterogeneous simulations depend largely on the inter-thresholds. If the inter-threshold values are sufficiently large, then Scenario 1-2 would very likely outperform Scenario 2-1.

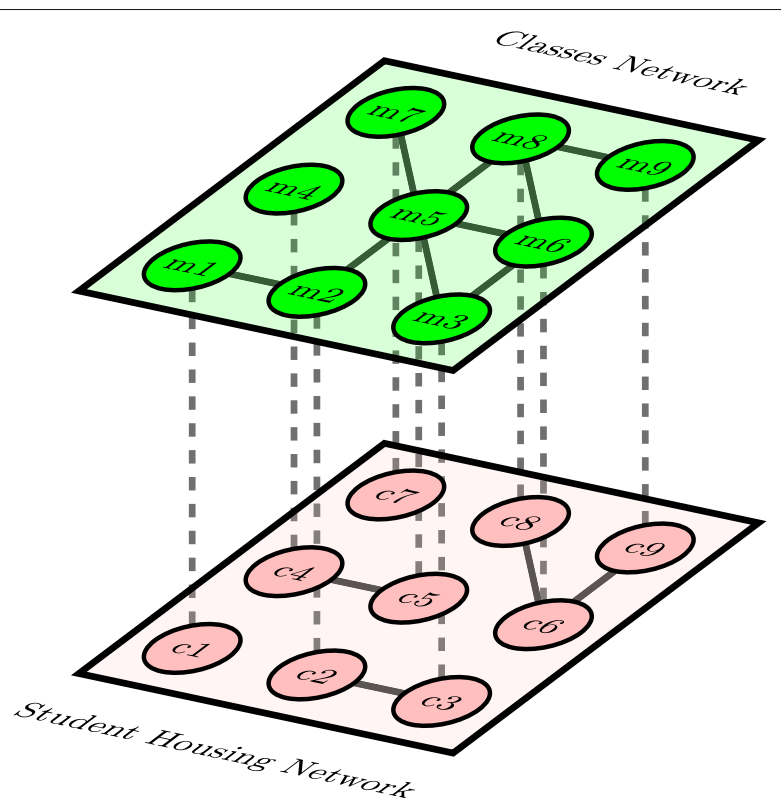

Fig. 4 Multiplex Network. An example of a two-layer multiplex social net- work where each node is represented in each layer. Each node $i_{A}$ has an inter- edge to node $i_{B}$ and each node $i_{B}$ has an inter-edge to node $i_{A}$. Layers "Classes Network" and "Student Housing Network" are social network layers where nodes (students) have edges to one another if they have a class together and if they live in the same student housing facility, respectively 


\section{Conclusions}

In closing, we studied how initial spreader selection strategies compare by analyzing the evolution of phenomena propagation over time using a state-of-the-art threshold-based propagation model. The benefit of our propagation model is that it is general enough to encapsulate other models of propagation (such as epidemic, linear probabilistic, linear threshold, etc.). Our results show that, if you have knowledge about the topological structure (such as degree of nodes), multi-layer designed selection outperforms singlelayer designed selection in most cases considered by our experiments. However, if you are in a situation where topological information is unavailable, random selection in a single-layer is more effective than multi-layer random selection. Additionally, from our work, we observe that single-layer selection by degree centrality (Scenario 2-1) generally outperforms two-layer selection at random (Scenario 1-2) most cases considered for this work.

Prior works investigating initial spreaders (or seed selection) in multi-layer networks are often interested in how phenomena propagation can be maximized or minimized given the context of the problem at hand (such as the influence maximization problem). In this work, our results are focused on analyzing how different interconnectivity cases and selection strategies with respect to layers considered for selection affect phenomena propagation. The general nature of our problem allows our work to provide insights to problems that aim to either maximize or minimize the overall affliction of a M-IDN topology as a result of phenomena propagation.

For future works in this direction, it is of interest to investigate the effectiveness of selection strategies in M-IDN topologies consisting of $m$ layers. With insight from how these varying strategies compare w.r.t. topological structure and interconnectivity, studying affliction in M-IDNs of $m$ layers may be easier to investigate.

\section{Endnotes}

${ }^{1}$ We do not consider random (or designed) single-layer spread with phenomena initially spreading in because it is essentially the same scenario, since these are general network components.

${ }^{2}$ https://github.com/khamfroush-lab/initial-spreaders-project

\section{Abbreviations}

BA: Barabási-Albert model for random graphs following a power law degree distribution; ER: Erdős-Rényi model for random graphs; ICM: Independent cascade model; M-IDN: Multi-layer interdependent network; PLC: Programmable logic controller; SCADA: Supervisory control and data acquisition; WS: Watts-Strogatz model for random, small-world graphs

\section{Acknowledgements}

Not applicable.

Authors' contributions

HK designed and formulated the problem, and proposed approaches to solve the problem described in this work. $\mathrm{NH}$ wrote the final draft of the paper for submission and analyzed results of experiments. SI ran simulations, documented results, and wrote an initial draft for the paper. MN collaborated with HK on formulation and simulation design and identifying the credibility of the problem formulation and simulations. All authors read and approved the final manuscript.

Funding

No outside funding was used to support this work. 
Ethics approval and consent to participate

Not applicable.

Consent for publication

Not applicable.

\section{Competing interests}

The authors declare that they have no competing interests.

\section{Author details}

${ }^{1}$ Department of Computer Science, University of Kentucky, University of Kentucky, Lexington 40506, KY, USA. ${ }^{2}$ Electrical Engineering Department, University of South Florida, E Fowler Ave, Tampa 33620, FL, USA.

Received: 6 November 2018 Accepted: 28 May 2019

Published online: 25 June 2019

\section{References}

Albert R, Jeong H, Barabási A-L (2000) Error and attack tolerance of complex networks. Nature 406(6794):378

Albright D, Brannan P, Walrond C (2010) Did Stuxnet Take Out 1,000 Centrifuges at the Natanz Enrichment Plant?. Institute for Science and International Security. https://isis-online.org/isis-reports/detail/did-stuxnet-take-out-1000centrifuges-at-the-natanz-enrichment-plant/

Anderson R, Fuloria S (2010) Who controls the off switch? In: 2010 First IEEE International Conference on Smart Grid Communications. IEEE, Gaithersburg. pp 96-101. https://doi.org/10.1109/SMARTGRID.2010.5622026. https:// ieeexplore.ieee.org/abstract/document/5622026

Barabási A-L, Albert R (1999) Emergence of scaling in random networks. Science 286(5439):509-512

Brooks R, Sander S, Deng J, Taiber J (2008) Automotive system security: challenges and state-of-the-art. In: Proceeding CSIIRW '08 Proceedings of the 4th annual workshop on Cyber security and information intelligence research: developing strategies to meet the cyber security and information intelligence challenges ahead. ACM, New York. p 26. https://dl.acm.org/citation.cfm?id=1413170

Charles M, Grinstead J, Snell L (1997) Introduction to probability. Am Math Soc. https://bookstore.ams.org/iprob/

Checkoway S, McCoy D, Kantor B, Anderson D, Shacham H, Savage S, Koscher K, Czeskis A, Roesner F, Kohno T, et al. (2011) Comprehensive experimental analyses of automotive attack surfaces. In: Proceeding SEC'11 Proceedings of the 20th USENIX conference on Security. USENIX, San Francisco. pp 77-92. https://dl.acm.org/citation.cfm?id=2028073

Chen W, Wang Y, Yang S (2009) Efficient influence maximization in social networks. In: Proceeding KDD '09 Proceedings of the 15th ACM SIGKDD international conference on Knowledge discovery and data mining. ACM, New York. pp 199-208. https://dl.acm.org/citation.cfm?id=1557047

Chen W, Wang C, Wang Y (2010) Scalable influence maximization for prevalent viral marketing in large-scale social networks. In: Proceeding KDD '10 Proceedings of the 16th ACM SIGKDD international conference on Knowledge discovery and data mining. ACM, New York. pp 1029-1038. https://dl.acm.org/citation.cfm?id=1835934

Cohen R, Erez K, Ben-Avraham D, Havlin S (2001) Breakdown of the internet under intentional attack. Phys Rev Lett 86(16):3682

Coleman J, Katz E, Menzel H (1957) The diffusion of an innovation among physicians. Sociometry 20(4):253-270

De Domenico M, Nicosia V, Arenas A, Latora V (2015) Structural reducibility of multilayer networks. Nat Commun 6:6864

Erdos P, Rényi A (1960) On the evolution of random graphs. Publ Math Inst Hung Acad Sci 5(1):17-60

Erlandsson F, Bródka P, Borg A (2017) Seed selection for information cascade in multilayer networks. arXiv.org 689 https://link.springer.com/chapter/10.1007/978-3-319-72150-7_35

Halperin D, Heydt-Benjamin TS, Fu K, Kohno T, Maisel WH (2008) Security and privacy for implantable medical devices. IEEE Pervasive Comput 7(1):30-39. https://ieeexplore.ieee.org/abstract/document/4431854

Hanna S, Rolles R, Molina-Markham A, Poosankam P, Blocki J, Fu K, Song D (2011) Take two software updates and see me in the morning: The case for software security evaluations of medical devices. In: Proceeding HealthSec'11 Proceedings of the 2nd USENIX conference on Health security and privacy. USENIX Association, Berkeley. https://dl. acm.org/citation.cfm?id=2028032

Hoppe T, Kiltz S, Dittmann J (2008) Security threats to automotive CAN networks-practical examples and selected short-term countermeasures. In: International Conference on Computer Safety, Reliability, and Security. Springer. pp 235-248. https://link.springer.com/chapter/10.1007/978-3-540-87698-4_21

Hudson N, Turner M, Nkansah A, Khamfroush H (2019) On the effectiveness of standard centrality metrics for interdependent networks. In: 2019 International Conference on Computing, Networking and Communications (ICNC). IEEE, Honolulu. https://ieeexplore.ieee.org/abstract/document/8685586

Khamfroush H, Bartolini N, La Porta TF, Swami A, Dillman J (2016) On propagation of phenomena in interdependent networks. IEEE Trans Netw Sci Eng 3(4):225-239

Kandhway K, Kuri J (2017) Using node centrality and optimal control to maximize information diffusion in social networks. IEEE Trans Syst Man Cybern Syst 47(7):1099-1110

Kempe D, Kleinberg J, Tardos É (2005) Influential nodes in a diffusion model for social networks. In: International Colloquium on Automata, Languages, and Programming. Springer. pp 1127-1138. https://link.springer.com/chapter/ 10.1007/11523468_91

Kim H, Beznosov K, Yoneki E (2014) Finding influential neighbors to maximize information diffusion in twitter. In: Proceeding WWW '14 Companion Proceedings of the 23rd International Conference on World Wide Web. ACM, New York. pp 701-706. https://doi.org/10.1145/2567948.2579358. https://dl.acm.org/citation.cfm?id=2579358

Kim, H, Beznosov K, Yoneki E (2015) A study on the influential neighbors to maximize information diffusion in online social networks. Comput Soc Networks 2(1):3 
Kimura M, Saito K, Motoda H (2009) Blocking links to minimize contamination spread in a social network. ACM Trans Knowl Discov Data (TKDD) 3(2):9

McDaniel P, McLaughlin S (2009) Security and privacy challenges in the smart grid. IEEE Secur Priv 7(3):75-77. https:// ieeexplore.iee. org/abstract/document/5054916

Metke AR, EkI RL (2010) Security technology for smart grid networks. IEEE Trans Smart Grid 1(1):99-107

Michalski R, Kajdanowicz T, Bródka P, Kazienko P (2014) Seed selection for spread of influence in social networks: Temporal vs. static approach. New Gener Comput 32(3-4):213-235

Mo Y, Kim TH-J, Brancik K, Dickinson D, Lee H, Perrig A, Sinopoli B (2012) Cyber-physical security of a smart grid infrastructure. Proc IEEE 100(1):195-209

Papanastasiou Y (2018) Fake news propagation and detection: A sequential model. https://papers.ssrn.com/sol3/Papers. cfm?abstract_id=3028354. Available at SSRN: https://ssrn.com/abstract=3028354 or https://doi.org/10.2139/ssm. 3028354

Pastor-Satorras R, Vespignani A (2001) Epidemic spreading in scale-free networks. Phys Rev Lett 86(14):3200

Rahman MA, Bera P, Al-Shaer E (2012) Smartanalyzer: A noninvasive security threat analyzer for ami smart grid. In: 2012 Proceedings IEEE INFOCOM. IEEE, Orlando. pp 2255-2263. https://ieeexplore.ieee.org/abstract/document/6195611. https://doi.org/10.1109/INFCOM.2012.6195611

Rushanan M, Rubin AD, Kune DF, Swanson CM (2014) Sok: Security and privacy in implantable medical devices and body area networks. In: 2014 IEEE Symposium on Security and Privacy. IEEE, San Jose. pp 524-539. https://ieeexplore.ieee. org/abstract/document/6956585. https://doi.org/10.1109/SP.2014.40

Salehi M, Sharma R, Marzolla M, Magnani M, Siyari P, Montesi D (2015) Spreading processes in multilayer networks. Netw Sci Eng IEEE Trans 2(2):65-83

Shu K, Bernard HR, Liu H (2019) Studying fake news via network analysis: detection and mitigation. In: Emerging Research Challenges and Opportunities in Computational Social Network Analysis and Mining. Springer. pp 43-65. https://link. springer.com/chapter/10.1007/978-3-319-94105-9_3

Stark C, Breitkreutz B-J, Reguly T, Boucher L, Breitkreutz A, Tyers M (2006) Biogrid: a general repository for interaction datasets. Nucleic Acids Res 34(suppl_1):535-539

Watts DJ, Strogatz SH (1998) Collective dynamics of 'small-world'networks. Nature 393(6684):440

Zhao D, Li L, Li S, Huo Y, Yang Y (2014) Identifying influential spreaders in interconnected networks. Phys Scr 89(1). https://iopscience.iop.org/article/10.1088/0031-8949/89/01/015203/meta

\section{Publisher's Note}

Springer Nature remains neutral with regard to jurisdictional claims in published maps and institutional affiliations.

\section{Submit your manuscript to a SpringerOpen ${ }^{\circ}$ journal and benefit from:}

- Convenient online submission

- Rigorous peer review

- Open access: articles freely available online

- High visibility within the field

- Retaining the copyright to your article

Submit your next manuscript at $\boldsymbol{\triangleright}$ springeropen.com 